\title{
Atrioventricular block (heart block) of the third degree
}

\author{
Melanie Humphreys, MA, BSc (Hon), ENB 124, RGN, ONC, Senior Lecturer, Post-registration Studies, \\ School of Health, New Cross Centre, University of Wolverhampton, Wolverhampton, UK
}

Senior Lecturer, Post-registration Studies, School of Health, New Cross Centre, University of Wolverhampton, Wolverhampton, UK. WV10 0QP. e-mail: in5277@wlv.ac.uk

\section{INTRODUCTION}

Atrioventricular (AV) block refers to the abnormality in electrical conduction between the atria and ventricles. It is also known as heart block. Atrioventricular block has different levels of severity (Wagner, 1994):

minor (first degree), in which all impulses are conducted with delay

moderate (second degree), in which some impulses fail to conduct

- complete (third degree), in which no impulses are conducted.

In adults, the most common causes of third-degree atrioventricular block are:

- acute myocardial infarction

- intoxication with drugs that affect the atrioventricular node (especially digitalis)

- chronic degeneration of the conduction pathway (Prosser et al., 2000; Smith and Kampine, 1990).

Occasionally, it occurs as a congenital anomaly, which may remain asymptomatic throughout life (Thompson, 1997).

Third-degree AV block divides the heart into two unconnected zones, which means that there is no relationship between the activity of the atria and of the ventricles. When no atrial impulses are conducted to the ventricles, the patient's clinical condition is determined by the escape capability of the more distal Purkinje cells. This escape capability is not necessarily stable.

Early detection and monitoring of this cardiac arrhythmia are essential in order to provide the appropriate nursing care.

\section{PHYSIOLOGY}

Rhythmic contraction of the heart relies on the organised propagation of electrical impulses along its conduction pathway. The marker of electrical stimulation is the action potential. This is created by a sequence of ion fluxes through specific channels in the cellular membranes. Cardiac cells capable of electrical excitation divide into three electrophysiological types (Lilly, 1993):

pacemaker cells (e.g. sinoatrial node, atrioventricular node) specialised, rapidly conducting tissues (e.g. Purkinje fibres)

- cardiac muscle cells.

Electrical activation of the heartbeat is normally initiated at the sinoatrial node. The wave of depolarisation rapidly spreads to the surrounding atrial muscle through intracellular gap junctions that provide electrical continuity between the cells. In general, the atrial muscle fibres participate in the propagation of the electrical impulse from the sinoatrial to the atrioventricular node. In certain regions, the fibres are more densely arranged, facilitating conduction (Tortora and Anagnostakos, 2000; Marriot and Condover, 1989).

In the normal conduction pathway, fibrous tissue surrounds the atrioventricular valves, separating the atrial from ventricular chambers. Thus, there is no electrical connection between the atria and ventricles other than through the atrioventricular node. As the electrical impulse reaches the atrioventricular node, there is a delay of approximately 0.1 seconds, as the small-diameter fibres in this region conduct slowly. This functional pause is beneficial because it allows the atria to empty their contents fully, before the impulse passes distally and provokes ventricular contraction (Humphreys, 2001a). In addition, the delay allows the atrioventricular node to serve as a 'gate-keeper' of conduction from the atria to ventricles. This function is critical for limiting the rate of ventricular stimulation during rapid atrial rhythms (Humphreys, 2001b).

After crossing the atrioventricular node, the cardiac action potential spreads into the rapidly conducting bundle of His, and into the right and left bundle branches. These divide into the Purkinje fibres, which radiate towards the myocardial fibres, stimulating them to contract in a synchronous and smooth way. This would be represented on the electrocardiograph (ECG) trace by the three major deflections:

- P wave (depolarisation of the atria)

- QRS complex (depolarisation of the ventricular muscle cells)

- T wave (repolarisation of the ventricular muscle cells).

In third-degree atrioventricular block, all impulses from the atria are blocked and none reach the ventricles. This is recognised on the ECG by the changing P to R relationship in the presence of a regular ventricular rhythm (Marriot and Condover, 1989). The 
block may occur above or below the bifurcation of the bundle of His. Regardless of the site of the block, the atria and ventricles beat independently, each being controlled by a separate pacemaker.

The sinoatrial node serves as the pacemaker for the atria, while the ventricles are governed by a ventricular focus at an intrinsic rate of 30 to 40 impulses per minute. Generally speaking, the lower the site for impulse generation of the ventricles, the slower the rate will be. For example, if the block is above the bifurcation of the His bundle, the ventricles may be stimulated by a junctional escape pacemaker at a rate of 40 to 60 impulses per minute.
However, in all cases, there is no relationship between the $\mathrm{P}$ waves and QRS complexes, known as atrioventricular dissociation. Furthermore, when atrioventricular block occurs in the lower parts of the His-Purkinje system, the ventricular escape complexes have wide QRS morphology (Wagner, 1994).

\section{ECG IDENTIFICATION}

The ECG abnormality is readily recognised, with total atrioventricular dissociation between the $\mathrm{P}$ waves and QRS complexes. Third-degree atrioventricular block may occur in the presence of any atrial rhythm (Box 1).

\section{BOX 1}

ECG showing third-degree atrioventricular (AV) block

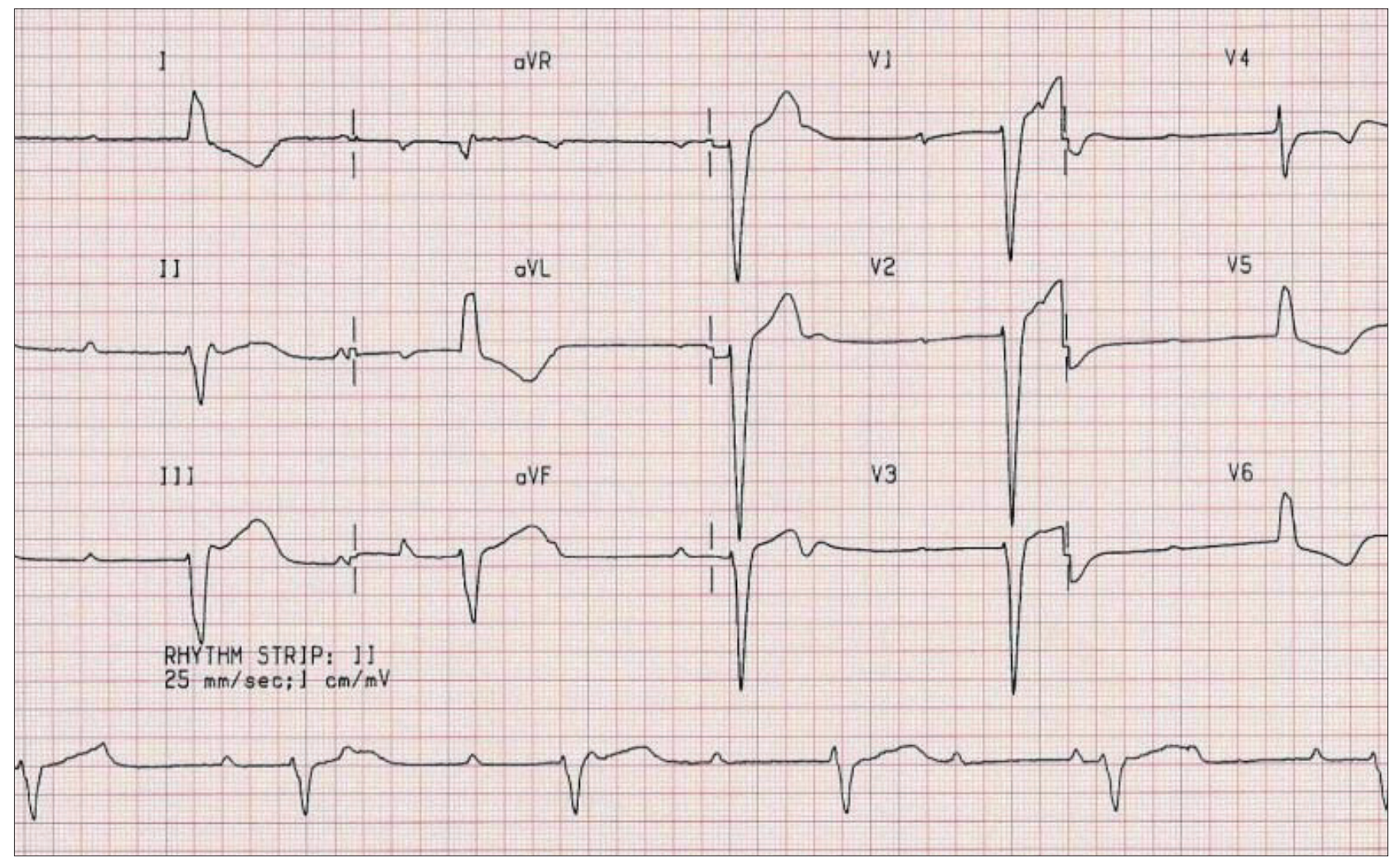

\section{TASK}

Undertake a systematic analysis of the following 12-lead ECG.

\section{COMMENTARY}

The 12-lead ECG shows the following features:

Heart rate, atrial: 70 bpm; ventricular: $30 \mathrm{bpm}$

- Rhythm: both P and QRS show regular patterns. However, there is no relationship between the two patterns (known as atrioventricular dissociation)

- P waves: normal

- PR interval: indeterminate due to dissociation

- QRS complex: $\mathrm{V} 1$ = wide $S$ wave with a positive $\mathrm{T}$ wave (preceded by small R wave); V6 = no initial Q wave, tall notched $\mathrm{R}$ wave with a negative $\mathrm{T}$ wave, which is consistent with a left bundle branch block (LBBB) pattern, denoting a right ventricular escape rhythm
QRS duration: 0.16 seconds. This is prolonged, which suggests that the impulse originated in the ventricles

- QT interval: 0.48 seconds. This is prolonged, which can be explained by a slow heart rate and prolonged depolarisation and repolarisation

- T wave: $\mathrm{V} 1-\mathrm{V} 3$ = elevated ST segments and upright $\mathrm{T}$ waves common in LBBB morphology; V5-V6 = depressed ST segment with inverted $\mathrm{T}$ waves, common in LBBB (secondary changes)

The diagnosis is third-degree atrioventricular block (also known as third-degree heart block), with a wide complex escape consistent with a LBBB pattern. This is synonymous with the escape rhythm originating in the ventricles.

This is an extremely dangerous arrhythmia presentation; it represents a clear warning of impending ventricular standstill or ventricular fibrillation (VF) (Resuscitation Council, 2000). 


\section{MANAGEMENT}

The clinical features depend primarily on the rate of the ventricular escape rhythm. However, because the ventricular rate is so slow, third-degree atrioventricular block presents a potentially life-threatening situation, as cardiac output can drop dramatically through the resultant reduction of frequency of ventricular ejection (Ambrose et al., 1997; Prosser et al., 2000). In addition, the patient loses his atrial kick which further decreases cardiac output (Humphreys, 2001a). This is a result of the loss of synchrony between the atrial and ventricular contractions.

Any exertion on the part of the patient can worsen symptoms, as the rate cannot increase to meet circulatory demands. Rates below 40 beats per minute are often associated with syncope or near-syncope, due to the gross reduction in blood flow to the brain. Confusion and light-headedness manifest lesser degrees of cerebral ischaemia. Rates of 40-60 beats per minute are associated with symptoms of weakness, faintness and lethargy. Often the rate of ventricular firing will be intermittent, producing an intermittent loss of consciousness (known as the Stokes-Adams syndrome) (Thompson, 1997). A few patients will be relatively free of symptoms, complaining only that they cannot tolerate exercise and that they are often tired for no apparent reason.

Although the severity of symptoms depends to a great extent on the resulting ventricular rate, an independent ventricular pacemaker is not dependable and may fail abruptly, causing ventricular standstill. Also, the slow ventricular rate predisposes to ventricular tachycardia (VT) or ventricular fibrillation (VF) by allowing more rapid foci in the ventricles to gain control of the heart rate (Dracup, 1995).

\section{Pacemakers}

Pacing is the only dependable method of treating third-degree atrioventricular block. This can be either transcutaneous, oeso- phageal or transvenous pacing, with the choice of modality being governed by the stability of the patient (Ambrose, 1988).

Both transcutaneous and oesophageal pacing are temporary measures, which are typically indicated for the unstable patient, as it buys time for the spontaneous recovery of the conducting system to occur or for more definitive treatment to be arranged. Pacing should be undertaken as soon as the block is identified.

Once transcutaneous pacing has resulted in an adequate cardiac output and the patient is stable, a transvenous pacing wire is usually inserted. While preparing for insertion of the pacemaker, an intravenous infusion of epinephrine (adrenaline), $2-10 \mu \mathrm{g} / \mathrm{min}$, should be considered, particularly if there are adverse signs and/or risk of asystole, which has not responded to atropine (Resuscitation Council, 2000). Alternative intravenous therapy would include isoproterenol (Isuprel), usually $1 \mathrm{mg}$ in 250 mL glucose solution (Dracup, 1995; Ambrose et al., 1997).

A temporary pacemaker will support the patient until the arrhythmia resolves, which is likely following an inferior-wall myocardial infarction, or until a permanent pacemaker has been inserted.

Common problems with pacemakers that can lead to low cardiac output and loss of AV synchrony include:

failure to capture

failure to pace

undersensing

oversensing.

In addition, malfunction of a pacemaker can lead to arrhythmias, hypotension, and syncope (Ambrose et al., 1997).

Failure to capture is probably the most common pacemaker problem. It is indicated on an ECG by a pacemaker spike without the appropriate atrial or ventricular response; this is a spike without a complex (Box 2). The pacemaker is unable to

\section{BOX 2}

ECG evidence of failure to capture - a common pacemaker problem

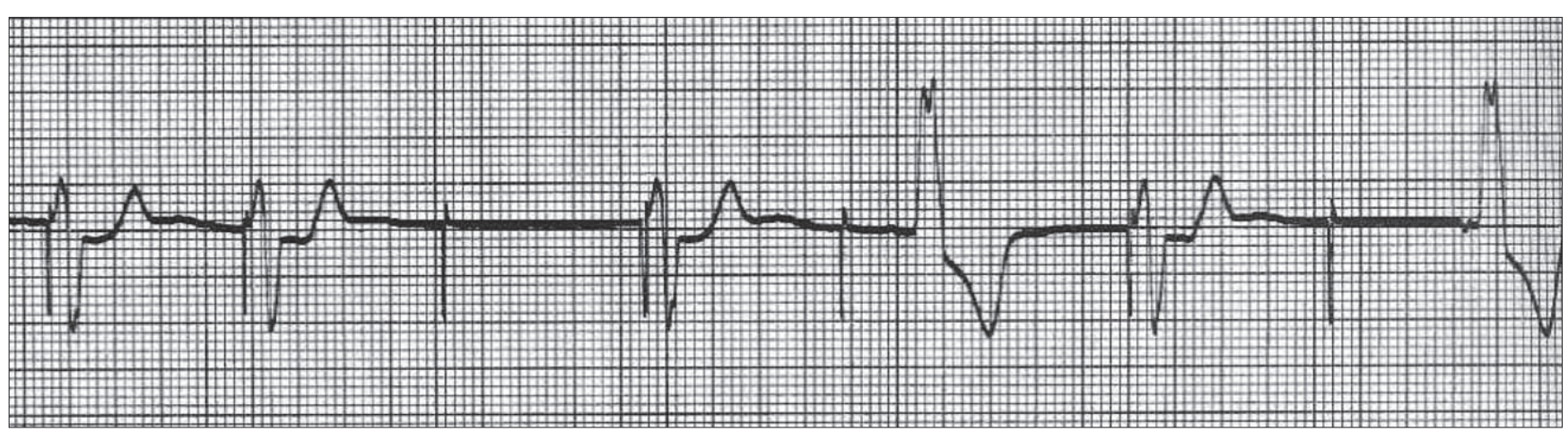

\section{TASK}

Consider the single-lead ECG recording in Box 2.

\section{COMMENTARY}

- Pacing spikes are evident at an automatic interval rate: 68 impulses per minute

- First two beats are paced (each QRS complex is preceded by a ventricular pacing spike)

- Third mark is a pacing spike; however, there is a failure to capture (no QRS complex evident)

- Fourth beat is a paced beat, followed by a failure-to- capture spike, patient beat (no prior pacing spike), paced beat, failure-to-capture spike, and finally another patient beat

- The complexes generated by pacing spike are normal ventricular beats, followed by a $\mathrm{T}$ wave

- The patient's own beats are ventricular with secondary T-wave changes (due to BBB pattern).

The concerning feature of this single-lead ECG is that there is evidence of frequent failure to capture of the pacemaker. It is essential to alert the patient's physician to this phenomenon. 
stimulate the chamber. Causes include acidosis, an electrolyte imbalance, fibrosis, an incorrect lead position, a low milliAmp (mA) setting, depletion of the battery, a cracked or broken leadwire, or perforation of the leadwire through the myocardium.

Patients with intermittent, or persistent, third-degree atrioventricular block causing symptoms need a planned permanent pacemaker. Implantation of a permanent pacemaker also improves the prognosis of patients with acquired third-degree atrioventricular block. It is therefore advisable, even in asymptomatic patients (Bloomfield and Miller, 1987).

\section{CONCLUSION}

Third-degree atrioventricular block is readily recognised from a 12-lead ECG recording. Clinically apparent haemodynamic consequences are common and include confusion, syncope and Stoke-Adams syndrome. Furthermore, the ventricular pacemaker is unreliable and may fail abruptly, causing the lifethreatening situation of ventricular standstill. Thus, early recognition and intervention are vital. The article has reviewed both the physiological processes of this important condition and the essential management strategy with the aim of promoting individualised care delivery.

\section{ACKNOWLEDGEMENT}

The author thanks Andrew Smallwood, Charge Nurse, CCU,
New Cross Hospital, Wolverhampton, UK, for his valued comments and reflections in the development of this paper.

\section{REFERENCES}

Ambrose C. (1988). Cardiac pacing: a new technique. The Professional Nurse; May: 290-291.

Ambrose ML, Mauro E, Nash J, Sinovic D. (1997). ECG Interpretation made Incredibly Easy! Pennsylvania: Springhouse.

Bloomfield P, Miller HC. (1987). Permanent pacing. British Medical Journal; 295: $741-744$.

Dracup K. (1995). Meltzer's Intensive Coronary Care: A Manual for Nurses. (5th ed.) California: Prentice-Hall International.

Humphreys M. (2001a). Atrial fibrillation: the most common form of arrhythmia. Connect Critical Nursing in Europe; 1(3): 93-96.

Humphreys M. (2001b). Patients with inferior MI: right ventricular involvement. Connect Critical Nursing in Europe; 1(2): 46-47.

Lilly LS. (1993). Pathophysiology of Heart Disease: A Collaborative Project of Medical Students and Faculty. London: Lea \& Febiger.

Marriot H, Condover MB. (1989). Advanced Concepts in Arrythmias. London: Mosby.

Prosser S, Worster B, MacGregor J, Dewar K. (2000). Applied Pharmacology. London: Mosby.

Resuscitation Council (UK). (2000). Advanced Life Support Course Provider Manual. (4th ed.) London: Resuscitation Council (UK) and European Resuscitation Council.

Smith JJ, Kampine JP. (1990). Circulatory Physiology - the Essentials. (3rd ed.) London: Williams \& Wilkins.

Thompson PL. (1997). Coronary Care Manual. London: Churchill Livingstone.

Tortora GJ, Anagnostakos NP. (2000). Principles of Anatomy and Physiology. (7th ed.) New York: Harper Collins.

Wagner GS. (1994). Marriott's Practical Electrocardiography. (9th ed.) London: Williams \& Wilkins.

\section{ON THE WEB}

Despite the red worm and many other deadly viruses across our globe, it is always nice to jump behind your computer on a cosy evening. And when you get bored with all the commercial web sites, just browse through some of the professional nursing and/or medical sites.

Every three months, hundreds of new web sites are developed. Many webmasters are hired to make the most attractive and accessible sites for companies and organisations, though there are a few web sites one can access only via email connection. In this issue, we have found a few sites that are very interesting for nursing colleagues in Europe, working in the field of intensive and critical care. Whether you are working on a neonatal, paediatric, or adult unit, there is always a site for your interest.

If you find a web site that you think might interest our readers, why don't you drop me a line? jos.latour@planet.nl

\section{http://groups.yahoo.com/group/PICU-Nurse-International}

This web site was established after the 3rd World Congress of Paediatric Intensive Care, held in Montreal, Canada, in June 2000. During a special forum meeting at the congress it was decided not to lose contact between so many active PICU nurses. The resulting web site is the hard work of our colleague Franco Carnevale.

The site is specially dedicated to a nursing group interested in discussing any matters related to paediatric intensive care. This group serves as a principal meeting place for the International Pediatric Intensive Care Nurses Network. Therefore, in addition to addressing specific clinical topics, this nursing group is also interested in discussing issues from an international perspective. Everyone can become a member of this group by just simply sending an e-mail.
In the past six months there have been about 60 to 100 discussions about various clinical nursing topics. So, if you have a problem with a child on the adult intensive care, just ask the international experts for help. Asking one question to the groups gives you loads of answers, and mostly within one hour.

\section{http://babelfish.altavista.com/tr}

Although most of the European countries have become one financial Europe since the introduction of the Euro, the matter of understanding each other remains difficult due to so many languages. Nevertheless, there is something nice on the web that can help our communication. The site mentioned above is a translation site. It is very helpful for 'Euro' nurses. Because you can copy and paste large amounts of text, it helps you to communicate and understand completely different languages. European languages included on the site are English, French, German, Italian, Portuguese, Spanish and Russian. And for those interested in translations from Japanese to English or visa versa, go ahead - give it a try! However, it is not perfect - and sometimes some very strange sentences are produced!

\section{http://WwW. frice.nl/}

In the first issue of CONNECT there was a web site presented about the Euricus-2 study. This web site is from the Foundation for Research on Intensive Care in Europe.

Just recently it has been have announced that the Euricus-3 study is finished and the full text is available on the web site. The Euricus-3 study is about the implementation of guidelines for budget control and cost calculation, and their effect on the quality of management of intensive care units in the countries of the European Union (EU).

Jos Latour 